\title{
Time-Scale Comovement Between The Indian And World Stock Markets
}

Rahul Deora, Indian Institute of Technology Kharagpur, India

Duc Khuong Nguyen, IPAG Lab, IPAG Business School, France

\begin{abstract}
We propose a wavelet-based dynamic conditional correlation - GARCH approach to investigate the time-scale comovement between the Indian and world stock markets. Our empirical analysis reveals the existence of time-scale-dependent comovement between Indian and world stock markets. The results can thus be used by heterogeneous groups of foreign and Indian investors who trade in different time horizons to actively manage and hedge against the risk of their portfolios.
\end{abstract}

Keywords: Comovement; Indian Stock Markets; DCC-GARCH; Wavelet Analysis

\section{INTRODUCTION}

C

nternational financial markets have become increasingly interdependent with the continued liberalization of cross-border capital flows. For global investors, there is a growing risk exposure relative to the asset price fluctuation over the world financial markets. In the context of modern portfolio theory, diversification benefits arise from negative and lower correlations between the assets included in the portfolio by an investor. Incentives for investing in international markets have been accentuated by lower return correlations between internationally-traded assets as compared with those between domestic assets (Grubel, 1968; Levy and Sarnat, 1970). As stock markets around the world have become more and more integrated, and especially within the developed market universe, global investors who seek international diversification benefit shifted, since the beginning of the 1980s, their attention to emerging markets. It is now well-known that these markets only have moderate correlations with their developed counterparts. However, efficient allocation strategies in emerging markets often require a careful analysis of their risk-return trade-off as well as their linkages with the world stock market.

Past studies have shown that expected returns in emerging markets vary over time under the effects of economic and financial forces as well as the degree of their integration with the world market (e.g., Bekaert and Harvey, 1995; Carrieri et al., 2007; Guesmi and Nguyen, 2011). Some recent studies find that emerging market returns may have time-scale behavior (e.g., Masih et al., 2010; Rua and Nunes, 2012; Graham et al., 2012). That is, stock returns may be characterized by multiscale structures, each occurs on a different time horizon owing to the presence of many types of market operators with heterogeneous risk preferences, capital budgeting constraints, information access, expectations, and risk perceptions. This new evidence thus suggests that an accurate modelling of the comovement between stock markets must consider the time scale effects.

The interest for wavelet method applications in economics and finance has increased in recent years (e.g., Ramsey and Lampart, 1998; Gencay et al., 2001, 2005; In and Kim, 2006; Sharkasi et al., 2006; Kim and In, 2007; Durai and Bhadurin, 2009). The majority of these studies show the usefulness of wavelet decomposition technique in modelling and apprehending the market risk and the relationships between stock returns and other economic variables. For example, Gençay et al. (2005) use wavelet analysis to investigate scaling properties of systematic risk as measured by the beta of an asset in a capital asset pricing model. The authors find that the relationship between the return of a portfolio and its beta becomes stronger as the scale increases. In other words, the predictive ability of the CAPM is more relevant at medium- to long-run horizons than at short-run horizons. Using a wavelet transform, Sharkasi et al. (2006) analyse the reaction of stock markets to crashes and events for a sample of both emerging and 
mature markets, and conclude that these markets respond differently to crashes. Kim and In (2007) study the relationship between stock returns and bond yields for the G7 countries, and find that the correlation between changes in stock prices and bond yields can differ from country to country and can also depend on the time scale.

While it is common that asset return correlations vary over time (e.g., De Santis and Gerard, 1997; Longin and Solnik, 2001), none of previous studies has looked at the possibility of changing cross-market correlations over different time horizons. In this article we fill in this gap by combining wavelet analysis with a bivariate DCCGARCH model of Engle (2002) to investigate the time-scale comovement between the Indian and world equity markets. Our empirical framework is motivated by the fact that international investors are heterogeneous in their trading strategies and each group of investors can operate on their own time horizon. As a result, stock market comovement in an international setting may differ across time scales.

At the empirical stage, we employ the discrete wavelet transform to decompose the original data into four different time horizons (2, 4, 8 and trading 16 days) as they help explaining almost all the dynamics of original return series. A bivariate DCC-GARCH model is then used to model the cross-market comovement. Using daily closing prices from January 17, 2007 to January 17, 2012 for the MSCI India Index and the MSCI World Stock Market Index, we find from our wavelet-based DCC-GARCH model that the comovement between the Indian and world equity markets vary considerably across time scales. Conventional DCC-GARCH estimate is thus only an "average" of the multiscale DCC-GARCH estimates. For almost all horizons, the GARCH coefficients are significant at the conventional levels, indicating strong evidence of time-varying volatilities at the decomposed levels as well as the usefulness of wavelet analysis. Furthermore, a lower estimated correlation between indices in a particular time horizon gives an indication that international investors should diversify their portfolio to accommodate the assets from the Indian emerging stock market in order to improve their portfolio's performance.

The remainder of the article is structured as follows. Section 2 describes the wavelet analysis and multiresolution decomposition. Section 3 introduces the DCC-GARCH models and its statistical properties. Section 4 describes the data used in the study and discusses the empirical findings. Finally, conclusions are given in Section 5.

\section{WAVELET ANALYSIS}

Wavelet theory is a powerful mathematical tool for time series analysis. Although its theoretical base lies in Fourier analysis, there are important differences between the wavelet theory and the theory based on Fourier analysis. While frequency content of the function is assumed to be stationary along the time axis in Fourier analysis, wavelets are, on the other hand, defined over a finite domain. Unlike the Fourier transform, they are localized both in time and in frequency. This feature makes them ideal candidates for analysing non-stationary signals and those with transients or singularities. They also have an advantage over traditional Fourier methods in analysing physical situations where the signals contain discontinuities and sharp spikes. The scale of resolution with which we look at the data plays an important role in wavelet analysis and the wavelets analyse data according to this scale of resolution. Looking at a signal at low-resolution scale only gives us an idea about its gross features, whereas looking at a higher resolution scale gives information about small features and details of the same data. Chui (1992), Daubechies (1992), and Percival and Walden (2000) provide a thorough review of wavelet analysis. Technical and practical applications of wavelet analysis are given in, among others, Gençay et al. (2002), and Bruce and Gao (1996).

\subsection{Wavelet decomposition}

Wavelets provide an ideal way to describe a signal contained in the underlying data. There are two types of wavelets defined on different normalization rules: father wavelets $\varphi$ and mother wavelets $\psi$. The father wavelet integrates to 1 and the mother wavelet integrates to 0 .

$$
\begin{aligned}
& \int \varphi(t) d t=1 \\
& \int \psi(t) \cdot d t=0
\end{aligned}
$$


The father wavelets are good at representing the smooth and low-frequency parts of a signal, and the mother wavelets are useful in describing the detail and high-frequency components. Any function $y(t)$ in $L^{2}(\mathbb{R})$ (space for square summable functions) to be represented by a wavelet analysis can be built up as a sequence of projections onto father and mother wavelets generated from $\varphi$ and $\psi$ through scaling and translation as follows

$\varphi_{j, k}(t)=2^{-j / 2} \varphi\left(2^{-j} t-k\right)=2^{-j / 2} \varphi\left(\frac{t-2^{j} k}{2^{j}}\right)$

$\psi_{j, k}(t)=2^{-j / 2} \psi\left(2^{-j} t-k\right)=2^{-j / 2} \psi\left(\frac{t-2^{j} k}{2^{j}}\right)$

They form a basis for functional analysis. The wavelet representation of the signal $y(t)$ in $L^{2}(\mathbb{R})$ can be written as:

$y(t)=\sum_{k} s_{J, k} \varphi_{J, k}(t)+\sum_{k} d_{J, k} \psi_{J, k}(t)+\sum_{k} d_{J-1, k} \psi_{J-1, k}(t)+\cdots+\sum_{k} d_{1, k} \psi_{1, k}(t)$

In the representation $\boldsymbol{J}$ is the number of multi-resolution components, and $s_{J, k}$ are called the smooth coefficients, and $d j, k$ are called the detailed coefficients. They are defined by:

$s_{J, k}=\int y(t) \varphi_{J, k}(t) d t$

$d_{J, k}=\int y(t) \psi_{J, k}(t) d t, \quad j=1,2 \ldots, J$

The magnitude of these coefficients reflects a measure of the contribution of the corresponding wavelet function to the total signal. The scale factor $2^{j}$ is also called the dilation factor, and the translation parameter $2^{\mathrm{j}} k$ refers to the location. For the larger the index $j$, the larger the scale factor $2^{j}$, and hence the function gets wider and more spread out. The translation parameter $2^{j} k$ is matched to the scale parameter $2^{j}$ in that as the functions $\varphi_{J, k}(t)$ and $\psi_{j, k}(t)$ get wider, their translation steps are correspondingly larger.

For multi-resolution decomposition, the decomposed signals are defined as follows:

$S_{j}(t)=\sum_{k} S_{J, k} \varphi_{J, k}(t)$

$D_{j}(t)=\sum_{k} d_{J, k} \psi_{j, k}(t) \quad$ for $j=1,2 . . J$

$S_{j}(t)$ and $\left.D_{j}(t)\right|_{j=1} ^{J}$ are called the smooth signal and the detail signals, respectively, which constitute a decomposition of a signal into orthogonal components at different scales. A signal $y(t)$ can thus be expressed in terms of these signals as:

$y(t)=S_{J}(t)+D_{J}(t)+D_{J-1}(t)+\cdots+D_{1}(t)$.

We can represent the discrete wavelet transform in matrix form. Let be the observation of length $T$. The string of wavelet coefficients can be ordered from fine scales to coarse scales as:

$w=\left(\begin{array}{c}d_{1} \\ d_{2} \\ \cdot \\ d_{J} \\ s_{J}\end{array}\right)$

Where $\mathrm{d}_{j}$ and $\mathrm{s}_{\mathrm{J}}$ are column vectors of the detailed coefficients $\left(\mathrm{d}_{\mathrm{j}, \mathrm{k}}\right)$ and $\left(\mathrm{s}_{\mathrm{J}, \mathrm{k}}\right)$ respectively. Taking the case that the sample size $T$ is divisible by $2^{J}$ for example, we have $T / 2$ coefficients at $d_{1, \mathrm{k}}$ (i.e., the finest scale), $T / 4$ at $d_{2, \mathrm{k}}$ (the next finest scale), and so forth until we find $T / 2^{J}$ coefficients for $d_{J, k}$ and $s_{J, k}$ (the coarsest scale), for a total amount of 
coefficients equal to $T=T / 2+T / 4+\cdots+T / 2^{J}+T / 2^{J}$. The number of coefficients is approximate if $T$ is not divisible by $2^{J}$.

The discrete wavelet transform (DWT) can then be represented by a matrix from as follows:

$\mathbf{w}=W y$,

Where $\mathrm{W}$ is a $(T \times T)$ real-valued orthonormal matrix defining the DWT which satisfies $\mathrm{W}^{T} \mathrm{~W}=I_{T}(T \times T$ identity matrix). We refer the details of $W$ on different wavelet bases and corresponding wavelet filter banks to Percival and Walden (2000). Using the DWT, we may formulate an additive decomposition of $\mathbf{y}$ by reconstructing the wavelet coefficients at each scale independently. Component $D_{j}$ can be represented as $D_{j}=\mathrm{W}_{j}^{T} \mathbf{w} j$ which define the $j^{\text {th }}$ level wavelet detail associated with changes in $\mathbf{y}$ at the scale $j$. The wavelet coefficients $\mathbf{w}_{j}=W_{j} \boldsymbol{y}$ represent the portion of the wavelet analysis (decomposition) attributable to scale $j$, while $\mathrm{W}_{j}^{T} \mathbf{w}_{j}$ is the portion of the wavelet synthesis (reconstruction) attributable to scale $j$.

\section{$2.2 \quad$ The choice of $\varphi, \psi$, and $J$}

For the choice of $\varphi$ and $\psi$, a traditional and popular wavelet function (or the daublet with length 8 , designated as "d8") is used for the study. Alternative choices for the basic wavelet such as "haar", "symmlet", and "coiflet" are tried for comparison, but the results are globally not much affected. For the multi-resolution level $J$, this study sets $J=4$ in the empirical analysis because decomposing raw data into four levels is matched with our convection in the investigation of spillover effects, namely, in daily, weekly, and monthly time horizons. Moreover, lower wavelet frequency components only account for very small variations in the original data. Hence, in our study, the highest frequency component $D 1$ represents short-term variations due to shocks occurring at a time scale of $2^{1}$ or 2 days (daily spillovers), and the next higher component $D_{2}$ accounts for variations at a time scale of $2^{2}$ or 4 days, that is near the working days of a week (weekly spillovers). Similarly, $D_{3}$ and $D_{4}$ components represent the midterm variations at time scale of $2^{3}$ or 8 and $2^{4}$ or 16 days (from weekly to monthly spillovers), respectively. $S_{4}$ is the smooth residual of the original signal after subtracting $D_{1}, D_{2}, D_{3}$, and $D_{4}$.

\section{DCC-GARCH MODEL AND TIME-SCALE COMOVEMENT MODELING}

The time-varying market comovement involving the MSCI World and Indian stock markets can be straightforwardly investigated on the basis of wavelet decomposition results. Empirical findings from this analysis are important as they are indicative of potential diversification benefits for global investors across time scales. The particular framework of the Dynamic Conditional Correlation - Generalized Autoregressive Conditional Heteroscedasticity (DCC-GARCH) model suggested by Engle (2002) thus provides a convenient way to address this issue. We are able to model not only the dynamic processes of conditional volatilities, but also the conditional correlations. Note that modelling and forecasting correlations between financial assets become a crucial need and multivariate GARCH models attract a growing interest at least for two main reasons. First, the availability of more and more powerful computers that enables the estimation of complex models with a high number of parameters. Second, this class of multivariate volatility models allows for both return and volatility interdependence, which is not possible with univariate models. The DCC-GARCH model of Engle (2002) is distinguished by its simplicity and flexibility when estimating a large variance-covariance matrix because the volatility of each return series composing the system can be modelled by a univariate GARCH specification. To date, several generalizations of the Engle (2002)'s model have been proposed (e.g., Cappiello and Engle, 2006; McAleer, 2005, Billio and Caporin, 2006). Prior research has adopted multivariate GARCH models to capture market information on the aggregate level (McAleer et al., 2009). In this article, we address the issue of time scale stock market comovement based on wavelet analysis.

Formally, the conditional variance-covariance matrix of the DCC-GARCH model can be written as:

$H_{t} \equiv D_{t} R_{t} D_{t}$ 
where $D_{t}=\operatorname{diag}\left\{\sqrt{ } h_{i t}\right\}$ is a $(2 \times 2)$ diagonal matrix of time-varying standard deviations estimated from univariate GARCH processes, and $R_{t} \equiv\left\{\rho_{i j}\right\}_{t}$ for $i, j=1$ and 2 , which is a correlation matrix containing conditional correlation coefficients. The elements in $D_{t}$ follow the univariate $\operatorname{GARCH}(p, q)$ processes in the following manner

$h_{i t}=\omega_{i}+\sum_{k=1}^{p} \alpha_{i} \varepsilon_{i t-k}^{2}+\sum_{k=1}^{q} \beta_{i} h_{i t-k} \quad \forall i=1,2$

The second component of the framework consists of a specific $\operatorname{DCC}(m, n)$ structure, which can be expressed as

$R_{t}=Q_{t}^{*-1} Q_{t} Q_{t}^{*-1}$,

with $Q_{t}=\left(1-\sum_{h=1}^{m} a_{h}-\sum_{l=1}^{n} b_{l}\right) \bar{Q}+\sum_{h=1}^{m} a_{h}\left(\varepsilon_{t-h} \varepsilon_{t-h}\right)+\sum_{l=1}^{n} b_{l} Q_{t-l}$

where $Q_{t}=\left\{q_{i j}\right\}_{t}$ is the conditional variance-covariance matrix of residuals with its time-invariant (unconditional) variance-covariance matrix $\bar{Q}$ obtained from estimating Eq. (2), and $Q_{t}^{*}=\operatorname{diag}\left\{\sqrt{ } q_{i t}\right\}$ is a (2×2) diagonal matrix containing the square root of the diagonal elements of $Q_{t} . a_{h}$ and $b_{l}$ are non-negative scalar parameter satisfying $a_{h}+b_{l}<1$. The key element of interest in $R_{t}$ is $\rho_{12, t}=q_{12, t} / \sqrt{q_{11, t} q_{22, t}}$ which represents the conditional correlation between nifty and commodity indices. Essentially, Eq. (3) relies on an autoregressive moving average type process to capture short-term deviations in the correlation around its long-run (unconditional) level.

\section{DATA AND EMPIRICAL RESULTS}

\subsection{Data description}

The data used in the study are the daily closing prices of the MSCI World stock market index and the Indian stock market index. All the data are obtained from Datastream International and encompass the period from January 17, 2007 to January 17, 2012. We have a total of 1305 observations in the sampling period. The continuously compounded returns are then computed using the following formula: $r_{t}=100 \times\left(\ln \left(P_{t}\right)-\ln \left(P_{t-1}\right)\right)$.

Table 1. Descriptive statistics of stock market returns

\begin{tabular}{lcc}
\hline & MSCI World & India \\
\hline Mean & -0.016 & -0.002 \\
Standard deviation & 1.405 & 2.187 \\
Minimum & -7.325 & -12.040 \\
Maximum & 9.096 & 19.490 \\
Skewness & -0.361 & 0.200 \\
Kurtosis & 8.684 & 6.933 \\
Q(5) & $23.362(0.000)$ & $6.454(0.265)$ \\
JB & $1783.5(<2.2 \mathrm{e}-16)$ & $2631.918(<2.2 \mathrm{e}-16)$ \\
ARCH(5) & $347.9147(<2.2 \mathrm{e}-16)$ & $46.390(7.566 \mathrm{e}-09)$ \\
\hline
\end{tabular}

Notes: Q(5), JB and ARCH(5) are the empirical statistics of the Ljung-Box test for serial correlation, the Jarque-Bera test for normality, and the Lagrange Multiplier (LM) test for conditional heteroscedasticity. The p-values associated with test statistics are in parentheses.

Selected descriptive statistics of daily returns are presented in Table 1. Over the study period, both the world and Indian stock markets realized negative average returns, -0.016 and -0.002 respectively. With reference to the standard deviation, we see that the Indian stock market is substantially more volatile than the world market. The Jarque-Bera tests reject the hypothesis of normality for both return series at the $1 \%$ level. The large values of the skewness and kurtosis coefficients further indicate that return distributions are typically asymmetric and leptokurtic. The Ljung-Box tests and the Lagrange Multiplier tests show strong evidence of return autocorrelation and ARCH effects for both return series. These stylized facts thus support our decision to combine the wavelet analysis with the DCC-GARCH model to apprehend the time scale comovement dynamics. The quasi-maximum likelihood estimation (QMLE) method is used to generate consistent estimates that are robust to non-normality. A comparison of the log-likelihood values among alternative lag-length specifications suggests that our data are best described by a $\operatorname{DCC}(1,1)$ with each of the conditional variances captured by a univariate $\operatorname{GARCH}(1,1)$ model, i.e., $p, q, m$, and $n$ 
are equal to 1 . This specification has also been widely used in the existing literature and is found to be suitable for modelling volatility dynamics and conditional correlations of stock returns (e.g., Lahrech and Sylwester, 2011).

\subsection{Empirical results}

We first apply a DCC(1,1)-GARCH $(1,1)$ model to the raw return data. The estimated coefficients are reported in Table 2 . They are highly significant at the $1 \%$ and $5 \%$ levels. The large values of the GARCH coefficients for the two corresponding univariate volatility processes $\left(\beta_{1}\right.$ and $\left.\beta_{2}\right)$ indicate that the conditional volatility of the world and Indian stock market returns depend much more on their past volatility than on past shocks to returns. The sum of $a_{l}$ and $b_{1}$ is lower than 1 , thus respecting the stability condition. The average correlation between world and Indian stock markets is not high (0.45). Fig. 3(a) shows the evolution of comovement between world and Indian stock markets the indices, $\rho_{12}$, when the data are unfiltered. It can be seen that the comovement index fluctuates significantly through time, reaches its highest level $(0.70)$ on June 2010. Although the results suggest substantial diversification benefits from investing in India, they are still not of helps for heterogeneous groups of investors on different trading horizons. This naturally motivates the investigation of short-, medium- and long-term periodic comovements.

As stated earlier, the major innovation of this paper lies in applying wavelet analysis to examine the complex multi-scale interactions between the world and Indian stock markets. The Daubechies least asymmetric filters with length 8 are applied to generate the multi-resolution decomposition of original return data. Stock market returns are thus decomposed into a trend series and four mutually orthogonal different periodicity (frequency) components, ranging from the shortest periodicity series to the longest-periodicity series or equivalently from the high frequency components ( 2 business days) to low frequency ones (16 business days). Our LM test reveals that the decomposed series is still exposed to conditional heteroscedasticity. The time-variations of the return series after decomposition are shown in Fig. 1 and Fig. 2 for world and Indian markets, respectively. We see that variations in stock returns are mainly caused by short-term fluctuations as most signal power concentrates on $D 1$ and $D 2$. In particular, the $D 1$ periodicity replicates almost all fluctuations in the original series. Such observed phenomenon seems to be consistent with the typical expectations that stock returns cannot be predicted in advance with ease, owing to their frequent and uncertain movements. It may thus be very complicated to implement an index investment strategy replicating the performance of the world and Indian stock market indices at the shortest horizon (2 business days).

Table 2. DCC-GARCH model estimation results on original series

\begin{tabular}{lc}
\hline Parameters & Estimates \\
\hline$\omega_{1}$ & $0.021^{*}(0.008)$ \\
$\omega_{2}$ & $0.063^{* * *}(0.021)$ \\
$\alpha_{1}$ & $0.096^{* *}(0.020)$ \\
$\alpha_{2}$ & $0.105^{* *}(0.032)$ \\
$\beta_{1}$ & $0.893^{* *}(0.024)$ \\
$\beta_{2}$ & $0.888^{* * *}(0.022)$ \\
$a_{1}$ & $0.059^{* * *}(0.011)$ \\
$b_{1}$ & $0.560^{*}(0.214)$ \\
Log-likelihood & -5600.127 \\
$\bar{\rho}_{12}$ & 0.451643 \\
\hline
\end{tabular}

Notes: $\omega_{1}, \alpha_{1}$, and $\beta_{1}$ are the estimates of the constant, ARCH and GARCH coefficients in the variance equation for the MSCI world market returns, respectively. $\omega_{2}, \alpha_{2}$, and $\beta_{2}$ are the estimates of the constant, ARCH and GARCH coefficients in the variance equation for the Indian market returns. Standard errors are indicated in parentheses. ${ }^{*}$ and ${ }^{* *}$ denote statistical significance at the $5 \%$ and $1 \%$ levels respectively. 
Figure 1. Daubechies-5 four-level wavelet decomposition of MSCI World market returns

The finest scale component $D 1$ represents short-term (or high frequency) variations due to shocks occurring at a time scale of $2^{1}$ $=2$ days, and the next finest component $D 2$ accounts for variations at a time scale of $2^{2}=4$ days, near the working days of a week. Similarly, $D 3$ and $D 4$ components represent the mid-term (weekly to monthly) variations at time scale of $2^{3}=8$ and $2^{4}=$ 16 days, respectively. $S 4$ is the residual of original signal after subtracting $D 1, D 2, D 3$, and $D 4$. The y-axis indicates the scale return in percentage.
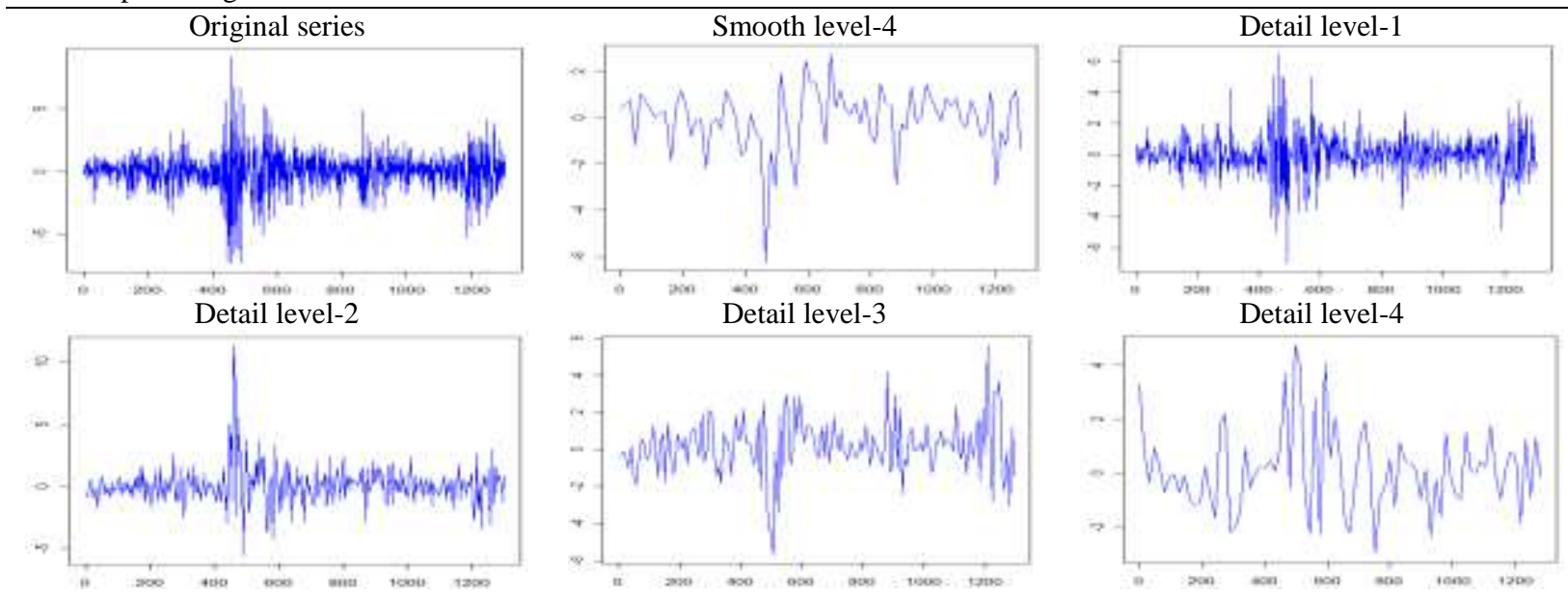

Figure 2. Daubechies-5 four-level wavelet decomposition of Indian stock market returns

The finest scale component $D 1$ represents short-term (or high frequency) variations due to shocks occurring at a time scale of $2^{1}$ $=2$ days, and the next finest component $D 2$ accounts for variations at a time scale of $2^{2}=4$ days, near the working days of a week. Similarly, $D 3$ and $D 4$ components represent the mid-term (weekly to monthly) variations at time scale of $2^{3}=8$ and $2^{4}=$ 16 days, respectively. $S 4$ is the residual of original signal after subtracting $D 1, D 2, D 3$, and $D 4$. The y-axis indicates the scale return in percentage.
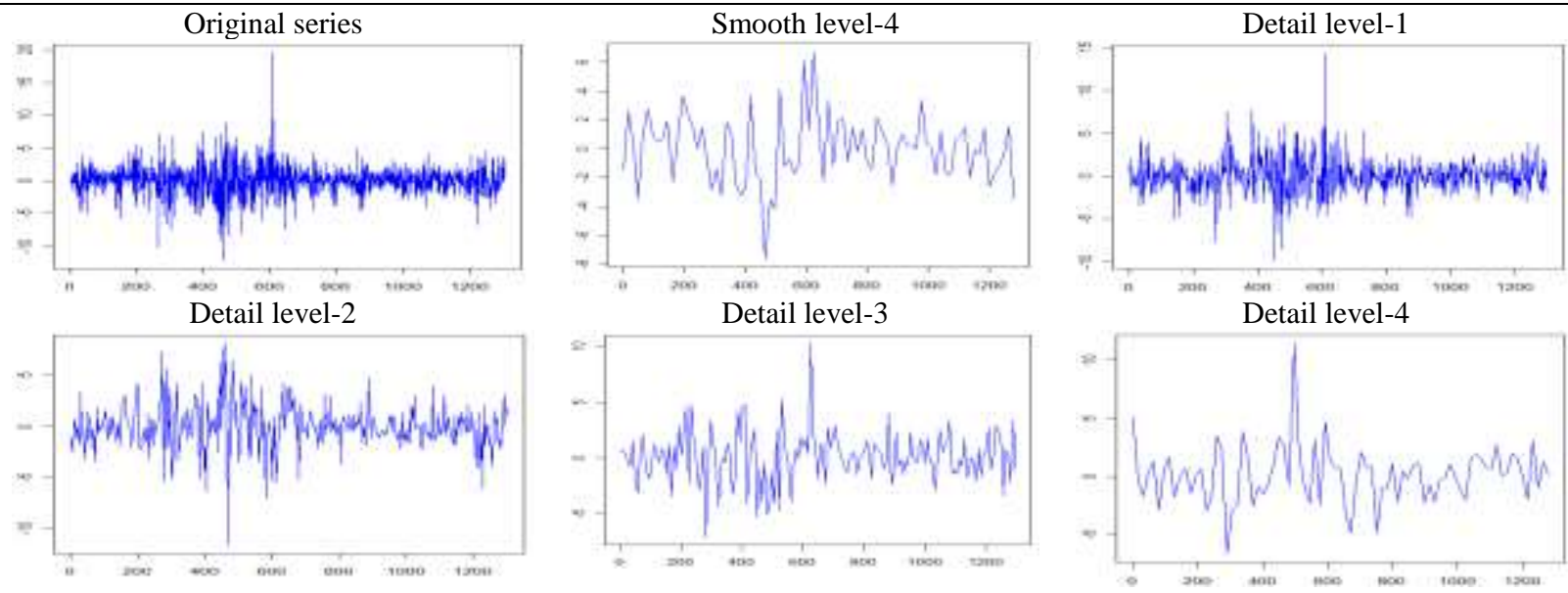

We report, in Table 3, summary statistics of decomposed returns together with time-scale risk-adjusted returns (Sharpe ratios). The estimated wavelet variance for both markets tends to increase when the scale increases. Stock returns in India also experience higher volatility than those on the world market index over the study period, whatever the scale considered. The Sharpe ratios reveal that the higher time-scale volatility in India is not always compensated with higher returns. 
Table 3. Summary statistics of decomposed returns

\begin{tabular}{lcccccccccc}
\hline & \multicolumn{4}{c}{ Indian stock market } & \multicolumn{5}{c}{ World stock market } \\
\hline & $D 1$ & $D 2$ & $D 3$ & $D 4$ & $S 4$ & $D 1$ & $D 2$ & $D 3$ & $D 4$ & $S 4$ \\
\hline Mean & 0.028 & -0.011 & 0.136 & 0.148 & -0.007 & -0.018 & 0.102 & 0.193 & 0.064 & -0.069 \\
Standard deviation & 2.067 & 2.268 & 2.267 & 2.503 & 2.352 & 1.290 & 1.539 & 1.518 & 1.535 & 1.359 \\
Maximum & 14.320 & 8.060 & 10.300 & 11.480 & 6.702 & 6.422 & 11.320 & 5.617 & 4.723 & 2.778 \\
Minimum & -9.847 & -11.600 & -7.060 & -6.689 & -7.613 & -6.956 & -5.567 & -5.676 & -3.003 & -6.246 \\
Sharpe ratio & 0.014 & -0.005 & 0.060 & 0.059 & -0.003 & -0.014 & 0.066 & 0.127 & 0.042 & -0.051 \\
\hline
\end{tabular}

While Table 2 reports the conventional DCC-GARCH estimates for the raw return data, Table 4 show the wavelet-based DCC-GARCH estimates for the two markets we consider over the different time scales. In view of the variance equation estimates, the DCC-GARCH model is still flexible enough to capture volatility and correlation dynamics of decomposed stock returns. Compared with the results for the raw returns, the unexpected parts of decomposed returns $\left(\alpha_{1}\right.$ and $\left.\alpha_{2}\right)$ no longer have significant effects on the current time-scale volatility, while the impacts from past time-scale volatility $\left(\beta_{1}\right.$, and $\left.\beta_{2}\right)$ are always large and highly significant. Taken together, we see that the degree of volatility persistence is lower than that of the raw data, but only past volatility matters for timescale volatility forecasting. More importantly, the average correlations between the world and Indian markets are found to be unevenly spread from the shortest-periodicity component to the longest-periodicity component, suggesting higher potential diversification gains for foreign investors in India at short-term investment horizons.

Table 4. Estimation results of DCC-GARCH model at different levels of decomposition

\begin{tabular}{|c|c|c|c|c|c|}
\hline Parameter & D1 & D2 & D3 & D4 & S4 \\
\hline \multirow{2}{*}{$\omega_{1}$} & $0.045^{*}$ & 0.088 & 0.298 & 0.598 & 0.352 \\
\hline & $(0.018)$ & $(0.046)$ & $(0.204)$ & $(0.608)$ & (1.027) \\
\hline \multirow[t]{2}{*}{$\omega_{2}$} & $0.188^{* * *}$ & $0.233^{*}$ & 0.138 & $0.413^{* *}$ & $0.382^{* *}$ \\
\hline & $(0.035)$ & $(0.089)$ & $(0.105)$ & $(0.130)$ & $(0.055)$ \\
\hline \multirow[t]{2}{*}{$\alpha_{1}$} & 0.168 & $0.236^{* *}$ & $0.285^{*}$ & 0.289 & 0.037 \\
\hline & $(0.358)$ & $(0.070)$ & $(0.135)$ & $(0.308)$ & $(0.535)$ \\
\hline \multirow[t]{2}{*}{$\alpha_{2}$} & 0.135 & 0.155 & 0.084 & 0.054 & 0.137 \\
\hline & $(0.090)$ & $(0.132)$ & $(0.266)$ & $(0.713)$ & $(0.437)$ \\
\hline \multirow[t]{2}{*}{$\beta_{1}$} & $0.810^{* *}$ & $0.735^{* *}$ & $0.595^{* *}$ & $0.447^{* *}$ & $0.776^{* *}$ \\
\hline & $(0.047)$ & $(0.056)$ & $(0.047)$ & $(0.036)$ & $(0.094)$ \\
\hline \multirow[t]{2}{*}{$\beta_{2}$} & $0.826^{* *}$ & $0.804^{* *}$ & $0.894^{* *}$ & $0.878^{* * *}$ & $0.795^{* *}$ \\
\hline & $(0.053)$ & $(0.060)$ & $(0.094)$ & $(0.143)$ & $(0.124)$ \\
\hline \multirow[t]{2}{*}{$a_{1}$} & 0.026 & $1.89 \mathrm{e}-08$ & 0.047 & 0.072 & $0.157^{* *}$ \\
\hline & $(0.016)$ & $(3.27 \mathrm{e}-02)$ & $(0.047)$ & $(0.048)$ & $(0.011)$ \\
\hline \multirow[t]{2}{*}{$b_{1}$} & $0.927^{* *}$ & 0.832 & $0.850^{* *}$ & $0.885^{* *}$ & $0.517^{*}$ \\
\hline & $(0.055)$ & $(4.80 \mathrm{e}-05)$ & $(0.190)$ & $(0.115)$ & $(0.246)$ \\
\hline \multirow{2}{*}{$\begin{array}{l}\text { Log-likelihood } \\
\bar{\rho}_{12}\end{array}$} & -2744.084 & -1481.25 & -778.0882 & -391.3542 & -374.6745 \\
\hline & 0.308162 & 0.452844 & 0.569995 & 0.802477 & 0.740812 \\
\hline
\end{tabular}

Notes: $\omega_{1}, \alpha_{1}$, and $\beta_{1}$ are the estimates of the constant, ARCH and GARCH coefficients in the variance equation for the MSCI world market returns, respectively. $\omega_{2}, \alpha_{2}$, and $\beta_{2}$ are the estimates of the constant, ARCH and GARCH coefficients in the variance equation for the Indian market returns. Standard errors are indicated in parentheses. ${ }^{*}$ and ${ }^{* *}$ denote statistical significance at the $5 \%$ and $1 \%$ levels respectively.

Time-varying World-India market correlations (comovement) are displayed in Fig. 3. We see that correlation coefficient averages at around 0.3 for level-1, while it is as high as 0.8 for the highest decomposition level. This phenomenon may be explained by the trading patterns of heterogeneous groups of investors. In the finest time scale, main traders are hedging strategists, speculators, and market makers. They trade in the international markets, accommodate the assets from the Indian market to diversify their portfolios, and thereby hedge against their risk. Using wavelet analysis for stock returns, In and Kim (2006) also observe that speculators and market makers intensively trade to realize a quick profit (or minimize a loss) over short time scales ranging from 1 to 2 trading days.

In the intermediate time scales ( $D 2$ and $D 3$ ), the main traders are international portfolio managers who mainly follow index tracking trading strategies (In and Kim, 2006). Accordingly, the stock trades typically occur on a weekly to monthly basis, with little attention paid to daily prices. Therefore, the variations in D2 and D3 
components (i.e., variations ranging from $2^{2}=4$ days to $2^{3}=8$ days) are no longer mean-reverting, but become persistent and tend to be more closely linked from one market to another. By this mechanism, the cross-market correlations increase when we move to the higher levels of decomposition. In the long-term trend components $D 4$ (time scale of $2^{4}=16$ days) and $S 4$, the main traders are central banks and buy-and-hold investors which operate on longer time horizons and often consider long-term economic fundamentals for their investment strategy. Consequently, $D 4$ and $S 4$ are more persistent than $D 2$ and $D 3$, and as a result the markets tend to comove much more together.

Figure 3. Conditional correlations between MSCI World and Indian stock markets at different time scales

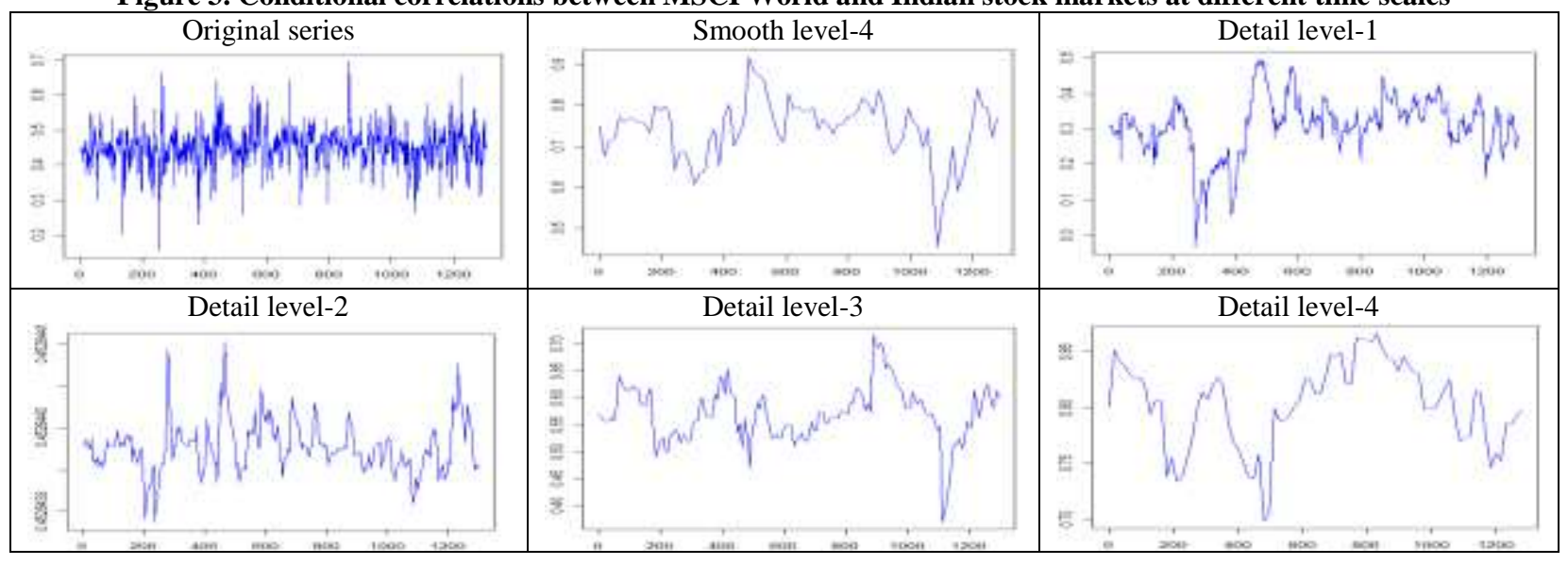

\section{CONCLUSION}

In this article, we combine the wavelet analysis and the DCC-GARCH model to analyse how much the Indian stock market moves together with the world market over the different time-scales. As far as stock markets are generally made of different groups of investors with different risk preferences and investment horizons, the results of our study can be used to implement good hedging strategies for simultaneous trading in the international markets. Unlike traditional multivariate GARCH models, the wavelet-based DCC-GARCH approach allows us to firstly decompose the raw returns into various sublevels according to various investment horizons practised by heterogeneous traders, and secondly study the detailed comovement dynamics at different frequencies.

Overall, our results indicate that stock returns in Indian and world stock markets have a time-varying scale behaviour and that the shortest periodicity components ( 2 business days) appears to reflect an important part of fluctuations in the original returns. More interestingly, both the direction and magnitude of comovement between the world and Indian markets vary greatly across the time scales used. As the level of the decomposition increases, the average correlation also increases between the two markets, thus suggesting a reduction of potential diversification benefits. For a daily international trader, accommodation of assets from Indian stock market would be beneficial in hedging the risk of their diversified portfolio. Daily traders and speculators in India can also be less worried about the spillovers from the developed international markets. The unique concern is the relatively higher risk of the shortest investment periodicity. As to international portfolio management institutions that trade in longer time horizons, they would be sceptical of investing in Indian markets due to higher correlations which exists at higher levels of decomposition, especially during periods of economic turbulences or crises. Our findings should also be of interest to speculators, hedge managers, international investors, as well as monetary and regulatory authorities, all of whom operate on very different time scales. Against this background, pinpointing the factors that contributed to the observed changes in the correlation of the indices would be an interesting subject for future research.

\section{AUTHOR INFORMATION}

Rahul Deora, Indian Institute of Technology Kharagpur, Kharagpur-721302, West Bengal, India. E-mail: rahuldeora91@gmail.com 
Duc Khuong Nguyen, IPAG Lab, IPAG Business School, 184 Boulevard Saint Germain, 75006 Paris, France. Phone: +33 153633600 E-mail: duc.nguyen@ipag.fr (Corresponding author)

\section{REFERENCES}

1. Billio, M., Caporin, M., Gobbo, M. (2006). Flexible Dynamic Conditional Correlation Multivariate GARCH for Asset Allocation. Applied Financial Economics Letters, 2(2) 123-130.

2. Bekaert, G., Harvey, C.R. (1995). Time-varying World Market Integration. Journal of Finance, 50(2) 403444.

3. $\quad$ Bruce, A., Gao, H.Y. (1996). Applied Wavelet Analysis with SPLUS. Springer-Verlag, New York.

4. Cappiello, L., Engle, R.F., Sheppard, K. (2006). Asymmetric Dynamics in the Correlations of Global Equity and Bond Returns. Journal of Financial Econometrics, 4(4) 537-572.

5. Carrieri, F., Errunza, V., Hogan, K. (2007). Characterizing World Market Integration through Time. Journal of Financial and Quantitative Analysis, 42(4) 915-940.

6. $\quad$ Chui, C.K. (2992). An Introduction to Wavelets. Academic Press, Boston, MA.

7. Daubechies, I. (1992). Ten Lectures on Wavelets. SIAM, Philadelphia, PA.

8. De Santis, G., Gerard, B. (1997). International Asset Pricing and Portfolio Diversification with Timevarying Risk. Journal of Finance, 52(5) 1881-1912.

9. Durai, S.R.S., Bhaduri, S.N. (2009). Stock Prices, Inflation and Output: Evidence from Wavelet Analysis. Economic Modelling, 26(5) 1089-1092.

10. Engle, R.F. (2002). Dynamic Conditional Correlation: A Simple Class of Multivariate Generalized Autoregressive Conditional Heteroskedasticity Models. Journal of Business and Economic Statistics, 20(3) 339-350.

11. Gençay, R., Selçuk, F., Whitcher, B. (2001). Scaling Properties of Foreign Exchange Volatility. Physica A: Statistical Mechanics and its Applications, 289(1-2) 249-266.

12. Gençay, R., Selçuk, F., Whitcher, B. (2002). An Introduction to Wavelets and Other Filtering Methods in Finance and Economics. Academic Press, London.

13. Gençay, R., Selçuk, F., Whitcher, B. (2005). Multiscale Systematic Risk. Journal of International Money and Finance, 24(1) 55-70.

14. Graham, M., Kiviaho, J., Nikkinen, J. (2012). Integration of 22 Emerging Stock Markets: A Threedimensional Analysis. Global Finance Journal, 23(1) 34-47.

15. Grubel, H.G. (1968). Internationally Diversified Portfolios: Welfare Gains and Capital Loss. American Economic Review, 58(5) 1299-1314.

16. Guesmi, K., Nguyen, D.K. (2011). How Strong is the Global Integration of Emerging Market Regions? An Empirical Assessment. Economic Modelling, 28(6) 2517-2527.

17. In, F., Kim, S. (2006). The Hedge Ratio and the Empirical Relationship between the Stock and Futures Markets: A New Approach using Wavelet Analysis. Journal of Business, 79(2) 799-820.

18. Kim, S., In, F. (2007). On the Relationship between Changes in Stock Prices and Bond Yields in the G7 Countries: Wavelet Analysis. Journal of International Financial Markets, Institutions and Money, 17(2) 167-179.

19. Lahrech, A., Sylwester, K. (2011). U.S. and Latin American Stock Market Linkages. Journal of International Money and Finance, 30(7) 1341-1357.

20. Levy, H., Sarnat, M. (1970). International Diversification of Investment Portfolios. American Economic Review, 60(4) 668-675.

21. Longin, F., Solnik, B. (2001). Extreme Correlation of International Equity Markets. Journal of Finance, 56(2) 646-676.

22. Masih, M., Alzahrani, M., Al-Titi, O. (2010). Systematic Risk and Time Scales: New Evidence from an Application of Wavelet Approach to the Emerging Gulf Stock Markets. International Review of Financial Analysis, 19(1) 10-18.

23. McAleer, M. (2005). Automated Inference and Learning in Modeling Financial Volatility. Econometric Theory, 21(1) 232-261.

24. McAleer, M., Hoti, S., Chan, F. (2009). Structure and Asymptotic Theory for Multivariate Asymmetric Conditional Volatility. Econometric Reviews, 28(5) 422-440. 
25. Percival, D., Walden, A. (2000). Wavelet Methods for Time Series Analysis. Cambridge University Press, Cambridge.

26. Ramsey, J.B., Lampart, C. (1998). Decomposition of Economic Relationships by Timescale using Wavelets. Macroeconomic Dynamics, 2(1) 49-71.

27. Rua, A., Nunes, L.C. (2012). A Wavelet-based Assessment of Market Risk: The Emerging Markets Case. Quarterly Review of Economics and Finance, 52(1) 84-92.

28. Sharkasi, A., Crane, M., Ruskin, H.J., Matos, J.A. (2006). The Reaction of Stock Markets to Crashes and Events: A Comparison Study between Emerging and Mature Markets using Wavelet Transforms. Physica A: Statistical Mechanics and its Applications, 368(2) 511-521. 


\section{$\underline{\text { NOTES }}$}

